\title{
Percutaneous sclerotherapy: recipe for perfect cooking
}

Josée Dubois

(C) Springer-Verlag 2011

Percutaneous sclerotherapy consists of a percutaneous injection of a chemical irritant into vein or lymphatic vessels to cause the destruction of the endothelium and the obliteration of the vascular space. In the literature, different sclerosing agents and injection protocols have been documented, with varying success and complications. Percutaneous sclerotherapy is a treatment modality for: (1) venous malformations, (2) lymphatic malformations and (3) aneurysmal bone cysts (as a treatment option). The main indications to treat venous malformations are pain, articular involvement and disfigurement. Lymphatic malformation sclerotherapy is indicated especially for disfigurement or mass effect. For aneurysmal bone cyst, treatment is indicated either by sclerotherapy and/or surgery.

\section{Sclerosing agents}

\section{Absolute ethanol}

Absolute ethanol (dehydrated alcohol, injection USP, 100\% v/v; Sandoz Canada Inc., Boucherville, QC) is the most destructive sclerosant and is assumed to have the lowest recurrence rate. It can be opacified with metrizamide powder or Ethiodol $(10 \mathrm{ml}$ ethanol: $2 \mathrm{ml}$ Ethiodol). The maximal dose per session should not exceed $1 \mathrm{ml} / \mathrm{kg}$; in children $0.5 \mathrm{ml} / \mathrm{kg}$ is recommended. The injection of ethanol induces a significant toxicity to the blood cells

Disclaimer Dr. Dubois has no financial interests, investigational or off-label uses to disclose.

J. Dubois $(\bowtie)$

CHU Sainte-Justine Mother and Child University Hospital Center, Montreal, Canada H3T 1C5

e-mail: josee-dubois@ssss.gouv.qc.ca and the intima, causing dehydration, protein denaturation, spasm and thrombosis, leading to a vascular obliteration. It produces marked tissue swelling because of intralesional thrombosis and edema. It is painful and, in most cases, necessitates general anesthesia.

Side effects Cutaneous necrosis, skin blistering and nerve palsy can be seen. Intravascular administration of ethanol can cause respiratory depression, cardiac arrhythmias, seizures, rhabdomyolysis, hypoglycemia and hemolysis, which can lead to hemoglobinuria and renal damage.

A less diffusible alcohol gel solution (ethylcelluloseethanol) was developed with interesting preliminary results although there are no large series reporting long-term efficacy.

\section{Detergent sclerosing agents}

Detergent sclerosing agents such as sodium tetradecyl sulfate (STS) (Thromboject 1\% and 3\%; Omega, Montreal, QC, Canada), polidecanol (Aethoxysclerol 1\%; Kreussler Pharma, Wiesbaden, Germany) and ethanolamine oleate (Ethamolin 5\%; Questcor Pharmaceuticals, Union City, CA, USA) cause endothelial injury by altering the surface tension surrounding the endothelial cells. STS is likely less toxic than ethanol because there are lower reported rates of skin necrosis and nerve impairment as well as fewer systemic complications. Since polidecanol has some anaesthetic properties, it is well tolerated during injection but its sclerosing efficacy could be less than STS and alcohol. Ethanolamine oleate is a salt of unsaturated fatty acid originally used in the treatment of gastroesophageal varices that induces thrombosis by damage to the vascular wall. Compared to ethanol, ethanolamine oleate has less effect on deeper layers of the vascular wall, as well as no penetrative 
effect, and is safer to use in situations where vascular structures are in nerves' proximity.

Side effects These materials can cause renal insufficiency, intravascular hemolysis, and hepatotoxicity. Anaphylactic reactions to STS injection and cardiac arrest after polidecanol injection have been reported.

Recently, a technique of foam sclerotherapy using a mix of one of these agents (STS, ethanolamine oleate or polidecanol) with lipiodol and air or carbon dioxide demonstrated better efficacy. The foam gives longer intimal contact and allows for a smaller dose of sclerosing agent. While the maximal dose is uncertain, the recommendation is to use no more than 10 $30 \mathrm{cc}$ and to repeat the sessions as needed.

\section{Doxycycline}

Doxycycline is a class of tetracycline antibiotic and comes as a lyophilized powder in a 100-mg bottle to which $5 \mathrm{cc}$ of contrast medium and $5 \mathrm{cc}$ of NACL serum $(10 \mathrm{mg} / \mathrm{ml})$ is added. In neonates, the maximum dose is generally $150 \mathrm{mg}$, while in older children it is $1,000 \mathrm{mg}$.

Side effects Hypoglycemia, metabolic acidosis and hemolytic anemia are the most often seen. Although yellow teeth discoloration has been reported, it was not specifically reported after intralesional injection.

\section{OK-432}

OK-432 (from Japan Picibanil) is a dead strain of group A streptococcus pyogenes incubated in penicillin. It is prepared by adding $10 \mathrm{ml}$ of saline to $0.1 \mathrm{mg}$ of OK-432 and has a maximum dose of $0.2 \mathrm{mg}$ per procedure. It is hypothesized that OK-432 causes a local cellular inflammatory reaction by either inducing apoptosis of the lymphatic endothelium or by stimulating the production of soluble cytokines.

Side effects They include fever, local inflammation, pain and swelling.

\section{Bleomycin}

Bleomycin is an antibiotic approved by the FDA as a chemotherapeutic agent. The dose is limited to $0.5 \mathrm{mg} / \mathrm{kg}$ or $15 \mathrm{mg}$ per session. It has been used in macrocystic and mixed malformations with a success rate varying from $70 \%$ to $95 \%$. Since it is administered locally, bleomycin is absorbed systemically at very low levels.
Side effects Fever is the most common side effect. Even in low doses, systemic bleomycin administration is suspected of being a causative agent in fatal pulmonary fibrosis and bone marrow suppression or immunosuppression. However, during local administration for lymphatic malformations, no definitive cases with these complications have been identified.

\section{Ethibloc}

Ethibloc is an alcoholic solution of zein (Ethnor Laboratories/Ethicon, Norderstedt, Germany). This product is not available at this time.

\section{Techniques and outcomes}

Vascular malformations

Direct punctures are performed with needles of variable gauges (20-, 22-, 24- or 25-gauge) under US guidance. When a free blood return is observed, direct venography using digital subtraction fluoroscopy (roadmap) or angiography is performed. The volume of contrast agent injected determines the sclerosant volume to be injected. Typically, the quantity of sclerosing agent equals the volume of contrast agent before obtaining opacification of a central vein. Injection is performed under fluoroscopic control and serial US examinations are useful to document the filling of the malformation by the sclerosant agent. Multiple punctures and injections are done to allow a complete filling of the lesion. In our experience, most treatments are repeated two to five times every 6 to 8 weeks depending of the lesion's volume. We believe multiple sessions with lower sclerosant injection volume could minimize complication occurrence. Success rates varying between $30 \%$ and $100 \%$ have been reported. The variations in sclerotherapy techniques and in criteria of success can explain this disparity. Most patients (80\%) had pain relief and lesion regression. On MRI, lesion regression can be modest even in the presence of symptom improvement.

Lymphatic malformations

Under US guidance, the cyst is first punctured with a 20- to 24-gauge needle. The lymphatic malformation is then emptied and finally injected with doxycycline or other agents. Some authors use a small pigtail catheter. The catheter is occluded, stopping the drainage for several hours, and is later re-opened. Repeated sclerotherapy is performed via this catheter over 3 consecutive days.

With doxycycline, OK-432 and bleomycin, the success rates for macrocystic lymphatic malformations are similar. 
Success has been reported in $60 \%$ to $87 \%$ of microcystic lymphatic malformations and $39 \%$ to $77 \%$ of mixed lymphatic malformations.

\section{Aneurysmal bone cyst}

Under fluoroscopy or CT-scan, the aneurysmal bone cyst is punctured with an 18-gauge arterial needle (Becton Dickinson and Co., Franklin Lakes, NJ, USA). When a CT-scan puncture is performed, we recommend transferring the patient to the angio suite to perform DSA acquisition to evaluate cyst volume and venous drainage. For many years, we used an alcohol solution of zein resulting in $94 \%$ excellent regression with complete bone re-ossification. However, this agent is not presently available. We are now using foam sclerotherapy with excellent preliminary results ( $80 \%$ of cases with documented regression). Most patients have only small residual cysts.

Complications are very rare. Pulmonary emboli and osteomyelitis are reported. Beware of ABCs close to the brain or spine; in these locations, we prefer using n-butyl cyanoacrylate (NBCA) to avoid migration of the sclerosing agent into the surrounding veins and subsequent central nervous injury.

The compilation and interpretation of results obtained via different agents (some authors mixed different sclerosing agents together), techniques and qualitative imaging outcomes pose many challenges. It is difficult to ascertain whether one agent is more effective than another. For most, the side effects as well as the complication rates are similar. 